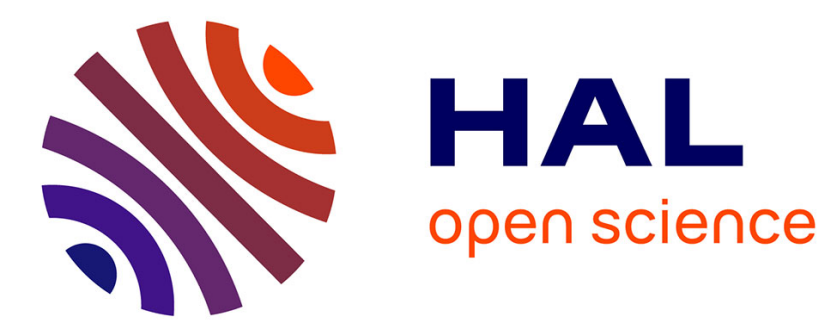

\title{
Cellular Automaton Models for Collective Cell Behaviour
}

\author{
Andreas Deutsch
}

\section{To cite this version:}

Andreas Deutsch. Cellular Automaton Models for Collective Cell Behaviour. 21th Workshop on Cellular Automata and Discrete Complex Systems (AUTOMATA), Jun 2015, Turku, Finland. pp.110, 10.1007/978-3-662-47221-7_1 . hal-01442478

\section{HAL Id: hal-01442478 https://hal.inria.fr/hal-01442478}

Submitted on 20 Jan 2017

HAL is a multi-disciplinary open access archive for the deposit and dissemination of scientific research documents, whether they are published or not. The documents may come from teaching and research institutions in France or abroad, or from public or private research centers.
L'archive ouverte pluridisciplinaire HAL, est destinée au dépôt et à la diffusion de documents scientifiques de niveau recherche, publiés ou non, émanant des établissements d'enseignement et de recherche français ou étrangers, des laboratoires publics ou privés. 


\title{
Cellular automaton models for collective cell behaviour
}

\author{
Andreas Deutsch \\ Cente for Information Services and High Performance Computing \\ Technische Universität Dresden
}

\section{Introduction}

Biological organisms are complex systems characterized by collective behaviour emerging out of the interaction of a large number of components (molecules and cells). In complex systems, even if the basic and local interactions are perfectly known, it is possible that the global (collective) behaviour can not be obviously extrapolated from the individual properties. Collective dynamics of migrating and interacting cell populations drive key processes in tissue formation and maintenance under normal and diseased conditions. For revealing the principles of tissue organization, it is fundamental to analyze the tissue-scale consequences of intercellular interaction [11,22]. Only an understanding of the dynamics of collective effects at the molecular and cellular scale allows answering biological key questions such as: what enables ensembles of molecules to organize themselves into cells? How do ensembles of cells create tissues and whole organisms? What is different in diseased tissues as malignant tumors? Mathematical models for spatio-temporal pattern formation can contribute to answer these questions. The first models of spatio-temporal pattern formation focused on the dynamics of diffusible morphogen signals and have been formulated as partial differential equations (e.g. [30]). In addition to diffusible molecular signals, the role of cells in morphogenesis can not be neglected. Living cells possess migration strategies that go beyond the merely random displacements of nonliving molecules (diffusion) [22]. More and more evidence exists about how the self-organization of interacting and migrating cells contributes to the formation of order in a developing organism. Thereby, both the particular type of cell interaction and migration are crucial and suitable combinations allow for a wide range of patterns. The question is: What are appropriate mathematical models for analyzing organization principles of moving and interacting cells cells?

Cellular automata (CA), in particular lattice-gas cellular automata (LGCA) can model the interplay of cells with themselves and their heterogeneous environment [17]. These models describe interactions at a cell-based local scale. Cell-based models (for a review see $[2,20]$ ) are required if one is attempting to extract the organization principles of interacting cell systems down to length scales of the order of a cell diameter to link the individual (microscopic) cell dynamics with a particular collective (macroscopic) phenomenon. 


\section{Cellular automata and lattice-gas cellular automata}

Cellular automaton models are a spatio-temporal modelling formalism and have been introduced by J. v. Neumann and S. Ulam in the 1950 s as a model of individual (self-)reproduction [12]. They consist of a regular spatial grid in which each grid point (or site) can have a finite, typically small number of discrete states. The next state of a site depends on the states in the neighboring sites and a next-state function, which can be deterministic or stochastic. Cellular automata provide simple models of self-organizing complex systems in which collective behaviour can emerge out of an ensemble of many interacting "simple" components - being it molecules, cells or organisms $[15,16,41]$.

Here, we suggest lattice-gas cellular automata which can be viewed as models for collective behaviour emerging from microscopic migration and interaction processes. LGCA can be used to model the interplay of cells with each other and with their heterogeneous environment by describing interactions at a cell-based (microscopic) scale and facilitating both efficient simulation and theoretical analysis of emergent, tissue-scale (macroscopic) parameters [17]. Historically, LGCA have been introduced as models of gas and fluid flows, through implementing simplistic local collisions. Often, the overall macroscopic behaviour of the system can be approximated very well if averages over larger spatial scales are considered $[23,40]$. In a biological context, LGCA particles are interpreted as cells and cell migration is modeled by updating cell positions at each time step based on local cell interactions. Local cell interactions are described by problem-specific LGCA transition rules. These transition rules are different from the rules that have been used for modelling fluid flows. LGCA transition rules in models of cell migration, in general, do not assume energy or momentum conservation. Biological LGCA models can be classified as stochastic cellular automata with time-discrete, synchronous updates consisting of stochastic interaction and subsequent deterministic migration steps. Implementing movement of individuals in traditional synchronous-update cellular automaton models is not straightforward, as one site in a lattice can typically only contain one individual, and consequently movement of individuals can cause collisions when two individuals want to move to the same empty site. In a lattice-gas model this problem is avoided by having separate channels for each direction of movement and imposing an exclusion principle. In addition, rest channels can be added for non-moving cells. The deterministic movement steps are alternated with stochastic interaction steps, in which processes affecting cell number, e.g., birth and death can be implemented (fig. 1).

A major advantage of LGCA models compared to other cell-based models for interacting cell systems, such as interacting particle systems, e.g. [29,39], asynchronous cellular automata, e.g. [4-6], further agent-based models [24] or systems of stochastic differential equations [37], is their computational efficiency. 
a) before interaction

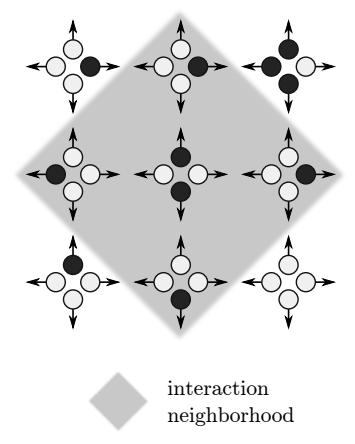

b) after interaction

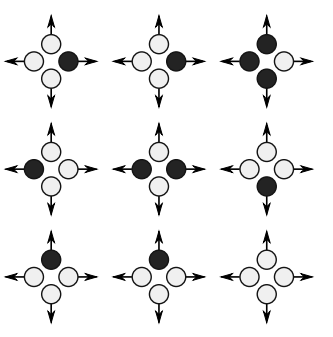

c) after migration

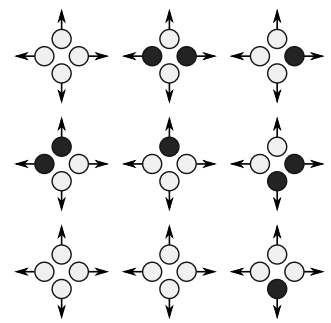

Fig. 1. Interaction and migration in a "biological LGCA": Filled circles denote occupied cells and open circles empty cells. a) part of the lattice before interaction, interation neighborhood is shown. b) after interaction: the configuration of the cells at several nodes has changed. c) after migration: each cell has moved to the nearest neighbor corresponding to its orientation indicated by arrows (the lattice outside the part shown is assumed to be empty, i.e. there is no migration of cells from outside).

\section{$3 \quad$ LGCA modelling and analysis}

The LGCA idea has led to models of morphogenetic motion describing migration of individual cells during spatio-temporal pattern formation in microorganisms, cell cultures and developing organisms $[7,17,35]^{1}$. The essential modelling idea is the definition of appropriate transition probabilities characterizing specific cell interactions. In particular, morphogenetic cell motion may be influenced by the interaction of cells with components of their immediate local surrounding through haptotaxis or differential adhesion, interaction with the basal lamina and the extracellular matrix, contact guidance, contact inhibition, and processes that involve cellular responses to signals that are propagated over larger distances (e.g. chemotaxis). LGCA models have also been used to study emergent collective behaviour in cell swarming [14], angiogenesis [32] and Turing pattern formation [19] (fig. 2). Moreover, there are methods for parameter estimation in LGCA models [31].

Deciphering the principles of collective cell behaviour is especially important for a better understanding of tumour growth and invasion and might allow to develop new therapy concepts. Besides more and more molecular investigations, mathematical modelling of selected aspects of tumour growth has become attractive within the last years (e.g. $[1,34,36])$. Cellular automaton models have

\footnotetext{
${ }^{1}$ A simulation platform containing various LGCA models with biological motivations can be found at www.biomodelling.info.
} 
a)

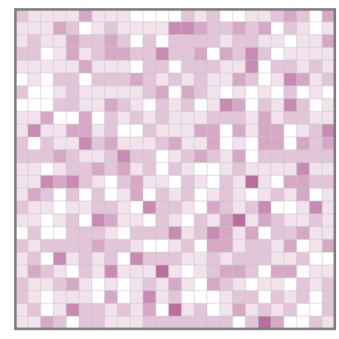

time 0

b)

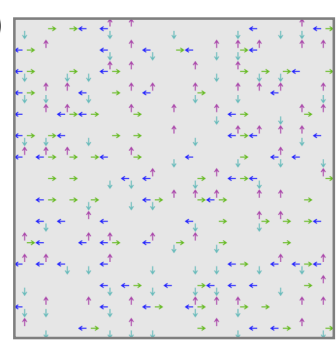

time 0

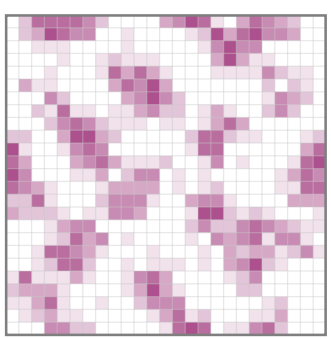

time 100

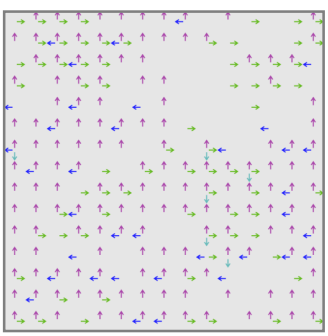

time 100

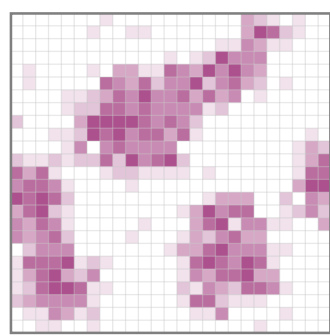

time 1000

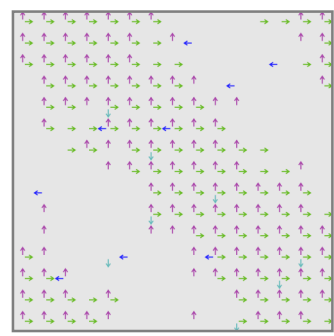

time 1000

Fig. 2. Spatio-temporal pattern formation in simulations (from a random initial cell population) for a) LGCA model of adhesive interaction, b) LGCA model of alignment interaction (see [14] and [17] for details). Colours indicate different cell numbers (a) and different cell directions (b).

been suggested for various aspects of tumour growth [34]. In particular, simulations and analysis of appropriate LGCA models permit to characterize different growth and invasion scenarios $[9,18,28]$ (fig. 3).

For a number of LGCA models a corresponding lattice-Boltzmann approximation can be adopted to analyze the emergence of spatio-temporal patterns [17]. The idea of the lattice-Boltzmann (mean-field) approximation is the reduction of the description of a system with many interacting individuals (many degrees of freedom), such as the CA, to the level of an effective, average description for the behaviour of a single individual (low degree of freedom). The application of the mean-field approximation allows for the transition from a microscopic to a macroscopic description of the CA dynamics. The central step is the derivation of a spatio-temporal mean-field approximation of the stochastic automaton process. Disregarding the spatial aspect completely leadd to qualitatively wrong model predictions (fig. 4). Based on a spatio-temporal mean-field description of the microscopic process, one can often calculate a corresponding macroscopic partial differential equation by means of a Chapman-Enskog expansion technique [16]. For example, from the mean-field PDE for a proliferation process one can derive important macroscopic observables of biological growth, such as total number of particles, per capita growth rate and invasion speed, and reveal their dependence on the microscopic growth and migration parameters [28]. 

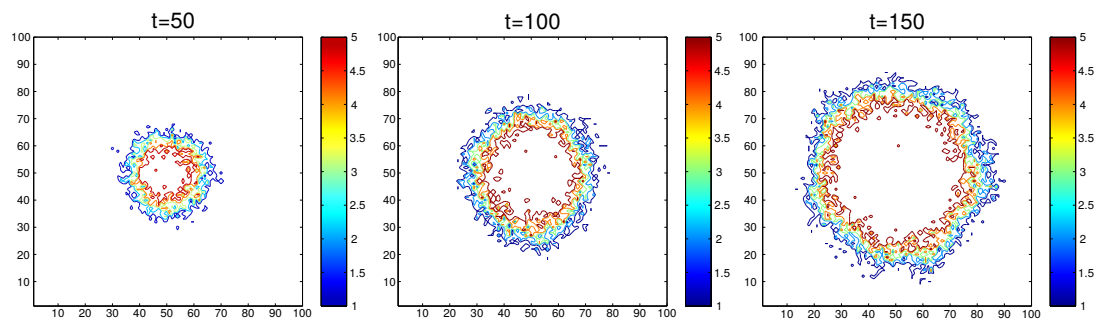

Fig. 3. Simulation of the spatio-temporal development of a LGCA growth model starting from a localized initial cell population in the centre of the lattice. The three pictures show simulation snapshots for subsequent time steps. Contour plot where colors indicate the cell density (from [27] with permission).

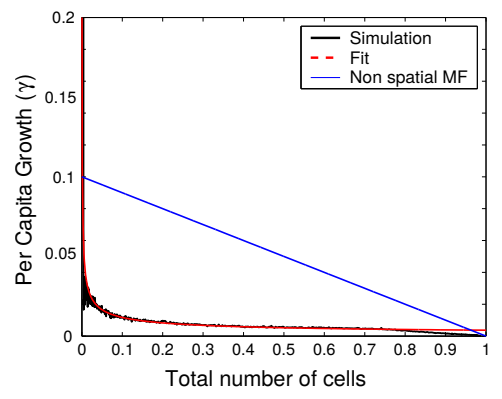

Fig. 4. LGCA growth model: evolution of the per capita growth rate as a function of the total population density. Comparison of simulations, a spatial mean-field approximation (fit) and a non-spatial mean-field approximation. The non-spatial mean-field approximation completely fails to describe the LGCA dynamics (from [27] with permission).

Typical examples for observables to study emergent collective behaviour are cell density patterns and related quantities such as the dynamics of moving cell fronts and cluster size distributions [8,26,32]. Cell density patterns can often be assessed experimentally and thus provide a means to relate LGCA model predictions to experimental observation. However, there are further experimental observables - particularly the trajectories of individual, selected cells - that characterize emergent collective behaviour but which are not directly deducible from cell density patterns. Classical LGCA, however, do not distinguish individual cells. We have developed an approach to overcome this limitation and which allows to translate classical LGCA into models where individual cells can be tracked (fig. 5). This is achieved by modifying the state space and the transition rules such that a cell identity can be transferred [33].

Based on these individual-based LGCA the establishment of a connection between the LGCA transition rules and a stochastic differential equation (SDE) description for the trajectories of single cells becomes possible. Comparable approaches to derive equations for tagged particles in cellular automaton models 

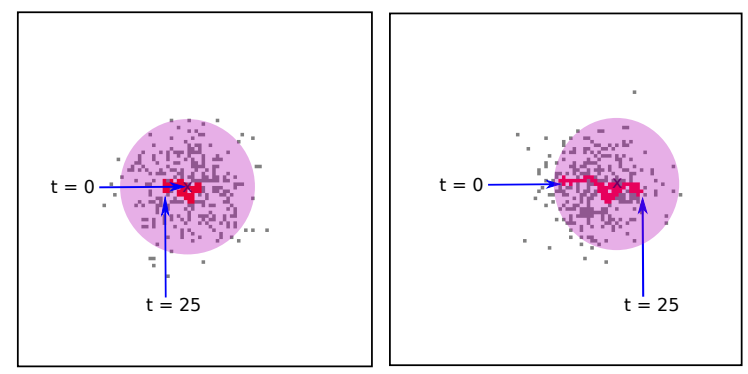

Fig. 5. LGCA simulations of single cell migration in a heterogeneous environment: Trajectories of selected labeled cells are shown in red. The positions of the labeled cells for $t=0$ and $t=25$ are marked by blue arrows. Simulation of the LGCA model for different sensitivities to the environment $\beta=0$ (A) and $\beta=0.5$ (B) (from [33] with permission).

$[3,25]$ are only applicable to equilibrium systems, i.e. where the cellular environment is in a steady state. Our approach allows to tackle LGCA models for non-equilibrium systems, i.e. the cellular environment can evolve over time [33]. For fairly general, cell number conserving LGCA transition rules, we have shown that the trajectories of individual cells can be described by an SDE whose parameters can be calculated directly from the LGCA transition rules. Cell number conserving LGCA transition rules are applicable when cell migration is on a much faster timescale than cell birth and death. The SDE description is derived in the limit when lattice spacing and time step length tend to zero under diffusive scaling. We have shown, for a variety of concrete models, that there is good agreement between LGCA simulations and the SDE approximation (fig. 6). Individual-based LGCA facilitate individual cell trajectory analysis while still allowing efficient simulations, thus opening up novel possibilities to investigate LGCA behaviour and to compare model and experiment at the individual cell level. Notice that the SDE approximation can be extended to other cell-based models such as interacting particle systems and general stochastic CA.

\section{Outlook}

The mean-field (Boltzmann) equation characterizing a given LGCA model arises under the assumption that the probability of finding two cells at specific positions is given by the product of corresponding single particle distribution functions, i.e. any correlations are neglected and distributions fully factorize. It is a challenge to include two-, three-, etc. particle distribution functions which will allow a systematic study of correlation effects. If pair correlations are taken into account, but third and higher order correlations are neglected, a generalized Boltzmann equation for the single particle distribution function is obtained, coupled to the so-called ring equation describing the evolution of the pair correlation function. For the adhesive (density-dependent) cellular automaton the ring equation has 


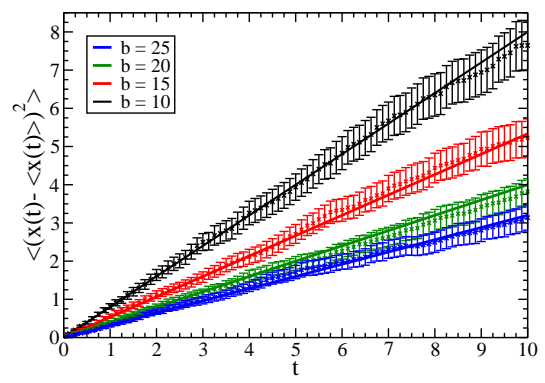

Fig. 6. Symmetric random walk in the low density regime. Simulations were carried out for a single cell on a two-dimensional square lattice lattice. Distribution $f(x)$ of the cell position of a single cell at time $t=10$. Time development of the mean square displacement of an individual cell. Simulation data (symbols) and corresponding solutions (lines) of stochastic differential equation for different numbers of rest channels are shown. All solutions were obtained for deterministic initial data. The simulation data were averaged over 500 independent simulations for each case. LGCA simulations and the corresponding SDE approximations show good agreement for the mean square displacement (from [33] with permission).

been successfully evaluated [13]. It is a challenge to determine corresponding equations for other cellular automata. This analysis could particularly improve our understanding of short and long time behaviour.

The need for discrete models, especially cellular automata, goes beyond the analysis of collective behaviour in interacting cell populations. A discrete celloriented approach is also required if the dynamic system behaviour depends on fluctuations at the individual cell level. This is, for example, the case at the front of invading tumours and crucial for the formation of metastases. Experimental findings of Bru et al. [10] indicate that many tumours share the same surface dynamics. This finding motivated the analysis of the tumour interface by means of a fractal scaling analysis [21].

In order to represent more detail at the individual cell level, multiscale models have been developed. For example, the user-friendly simulation platform Morpheus integrates cell-based models, ordinary differential equations for subcellular dynamics and reaction-diffusion systems for the extracellular environment [38]. While there exists a rich theory on ordinary and partial differential equations, the theory for cell-based models, in particular cellular automata is comparably young. Based on the variability in the local dynamics, we demonstrated that the "interaction-modul oriented" cellular automaton modelling provides an intuitive and powerful approach to capture essential aspects of collective cellular dynamics [17]. In conclusion, there are both challenging future perspectives with regards to interesting biological applications of the lattice-gas cellular automaton idea and possible refinements of analytical tools for the investigation of lattice-gas 
cellular automata. Hopefully, the potential of cellular automata for modelling essential aspects of biological systems will be further exploited in the future.

\section{Acknowledgments}

AD acknowledges the support of the German Research Foundation (DFG) within the Cluster of Excellence "Center for Advancing Electronics Dresden" and thanks

H. Hatzikirou, C. Mente, R. Müller and J. Starruss (Dresden) for comments and discussions.

\section{References}

1. Anderson, A.R.A., Quaranta, V.: Integrative mathematical oncology. Nature Rev. Cancer 8, 227-234 (2008)

2. Anderson, A., Chaplain, M., Rejniak, K. (eds.): Single cell-based models in biology and medicine. Birkhauser, Basel (2007)

3. Arratia, R.: The motion of a tagged particle in the simple symmetric exclusion system on Z. Ann. Prob. 11, 362-373 (1983)

4. Badoual, M., Deroulers, C., Aubert, M., Grammaticos, B.: Modelling intercellular communication and its effects on tumour invasion. Phys. Biol. 7(4), 046013 (2010)

5. Binder, B.J., Landman, K.A., Newgreen, D.F., Simkin, J.E., Takahashi, Y., Zhang, D.: Spatial analysis of multi-species exclusion processes: application to neural crest cell migration in the embryonic gut. Bull. Math. Biol. 74(2), 474-490 (2012)

6. Bloomfield, J.M., Sherratt, J.A., Painter, K.J., Landini, G.: Cellular automata and integro-differential equation models for cell renewal in mosaic tissues. J. R. Soc. Interface 7(52), 1525-1535 (2010)

7. Börner, U., Deutsch, A., Bär, M.: A generalized discrete model linking rippling pattern formation and individual cell reversal statistics in colonies of myxobacteria. Phys. Biol. 3(2), 138-146 (2006)

8. Böttger, K., Hatzikirou, H., Chauviere, A., Deutsch, A.: Investigation of the migration/proliferation dichotomy and its impact on avascular glioma invasion. Math. Model. Nat. Phenom. 7(1), 105-135 (2012)

9. Böttger, K., Hatzikirou, H., Voss-Böhme, A., Cavalcanti-Adam, E.A., Herrero, M.A., Deutsch, A.: Emerging Allee effect in tumor growth. Plos. Comp. Bio., in press (2015)

10. Brú, A., Albertos, S., Subiza, J.L., López García-Asenjo, J.A., Brú, I.: The universal dynamics of tumor growth. Biophys. J. 85, 294861 (2003)

11. Bryant, D.M., Mostov, K.E.: From cells to organs: building polarized tissue. Nature Rev. Mol. Cell Biol. 9, 887-901 (2008)

12. Burks, A.W.: Essays on Cellular Automata. University of Illinois Press, Urbana IL (1970)

13. Bussemaker, H.: Analysis of a pattern-forming lattice-gas automaton: mean field theory and beyond. Phys. Rev. E 53, 1644-1661 (1996)

14. Bussemaker, H.J., Deutsch, A., Geigant, E.: Mean-field analysis of a dynamical phase transition in a cellular automaton model for collective motion. Phys. Rev. Lett. 78, 5018-5021 (1997)

15. Casti, J.L.: Alternate realities. John Wiley, New York (1989) 
16. Chopard, B., Droz, M.: Cellular automata modeling of physical systems. Cambridge University Press, New York (1998)

17. Deutsch, A., Dormann, S.: Cellular automaton modeling of biological pattern formation: characterization, applications, and analysis. Birkhauser, Boston (2005 (2nd ed 2015))

18. Dormann, S., Deutsch, A.: Modeling of self-organized avascular tumor growth with a hybrid cellular automaton. In Silico Biol. 2(3), 393-406 (2002)

19. Dormann, S., Deutsch, A., Lawniczak, A.T.: Fourier analysis of Turing-like pattern formation in cellular automaton models. Future Gener. Comp. Sy. 17(7), $901-909$ (2001)

20. Drasdo, D.: On selected individual-based approaches to the dynamics in multicellular systems. In: Alt, W., Chaplain, M., Griebel, M., Lenz, J. (eds.) Models of polymer and cell dynamics. Birkhäuser, Basel (2003)

21. de Franciscis, S., Hatzikirou, H., Deutsch, A.: Analysis of lattice-gas models for tumor growth by means of fractal scaling. Acta Phys. Pol. B., Proc. Suppl. 2(4), $167(2011)$

22. Friedl, P., Gilmour, D.: Collective cell migration in morphogenesis, regeneration and cancer. Nature Rev. Mol. Cell Biol. 10, 445-457 (2009)

23. Frisch, U., Hasslacher, B., Pomeau, Y.: Lattice-gas automata for the Navier-Stokes equation. Phys. Rev. Lett. 56(14), 1505-1508 (1986)

24. Galle, J., Hoffmann, M., Aust, G.: From single cells to tissue architecturea bottomup approach to modelling the spatio-temporal organisation of complex multicellular systems. J. Math. Biol. 58, 261-283 (2009)

25. Harris, T.E.: Diffusion with collisions between particles. J. App. Prob. 2, 323-338 (1965)

26. Hatzikirou, H., Basanta, D., Simon, M., Schaller, K., Deutsch, A.: Go or grow: the key to the emergence of invasion in tumour progression? Math. Med. Biol. 29(1), 49-65 (2006)

27. Hatzikirou, H., Brusch, L., Schaller, C., Simon, M., Deutsch, A.: Prediction of traveling front behavior in a lattice-gas cellular automaton model for tumor invasion. Comput. Math. Appl. 59(7), 2326 - 2339 (2010)

28. Hatzikirou, H., Deutsch, A.: Cellular automaton modelling of tumor invasion. In: Meyers, R. (ed.) Encyclopedia of complexity and systems science. Springer, New York (2009)

29. Liggett, T.M.: Interacting particle systems. Springer (1985)

30. Meinhardt, H.: Models of biological pattern formation. Academic Press, London (1982)

31. Mente, C., Prade, I., Brusch, L., Breier, G., Deutsch, A.: Parameter estimation with a novel gradient-based optimization method for biological lattice-gas cellular automaton models. J. Math. Biol. 63(1), 173-200 (2010)

32. Mente, C., Prade, I., Brusch, L., Breier, G., Deutsch, A.: A lattice-gas cellular automaton model for in vitro sprouting angiogenesis. Acta Phys. Pol. B 5(1), 99$115(2012)$

33. Mente, C., Voss-Böhme, A., Deutsch, A.: Analysis of individual cell trajectories in lattice-gas cellular automaton models for migrating cell populations. Bull. Math. Biol. (2015, to appear)

34. Moreira, J., Deutsch, A.: Cellular automaton models of tumour development - a critical review. Adv. Compl Syst. (ACS) 5(2), 1-21 (2002)

35. Moreira, J., Deutsch, A.: Pigment pattern formation in zebrafish during late larval stages: A model based on local interactions. Developm. Dyn. 232(1), 33-42 (2004) 
36. Preziosi, L. (ed.): Cancer modelling and simulation. Chapman Hall/CRC Press, Boca Raton, Florida, USA (2003)

37. Rejniak, K.A., Anderson, A.R.A.: Hybrid models of tumor growth. Wiley Interdiscip. Rev. Syst. Biol. Med. 3(1), 115-125 (2011)

38. Starruß, J., de Back, W., Brusch, L., Deutsch, A.: Morpheus: a user-friendly modeling environment for multiscale and multicellular systems biology. Bioinformatics 30, 1331-1332 (2014)

39. Voss-Böhme, A., Deutsch, A.: The cellular basis of cell sorting kinetics. J. Theor. Biol. 263(4), 419-436 (2010)

40. Wolf-Gladrow, D.A.: Lattice-gas cellular automata and lattice Boltzmann models. Springer, Berlin (2000)

41. Wolfram, S.: A new kind of science. Wolfram Media, Inc (2002) 